\title{
IN VIVO METHODS TO STUDY UPTAKE OF NANOPARTICLES INTO THE BRAIN
}

\author{
Kaur Harpreet*, Singh Gurpreet \\ Rayat Institute of Pharmacy,SBS Nagar, Ropar,Punjab-140001,India \\ *Corresponding Author's E-mail: kaurkhalsa1988@yahoo.in
}

\begin{abstract}
ABTRACT
Several in vivo techniques have been developed to study and measure the uptake of CNS compounds into the brain. With these techniques various parameters can be determined after drug administration, including the blood to brain influx constant $\left(\mathrm{K}_{\text {in }}\right)$, the permeability-surface area (PS) product, and the brain uptake index (BUI). These techniques have been mostly used for drugs that are expected to enter the brain via transmembrane diffusion or by carrier-mediated transcytosis. Drugs that have limitations in entering the brain via such pathways have been encapsulated in nanoparticles (based on lipids or synthetic polymers) to enhance brain uptake. Nanoparticles are different from CNS compounds in size, composition and uptake mechanisms. This has led to different methods and approaches to study brain uptake in vivo. Here we discuss the techniques generally used to measure nanoparticle uptake in addition to the techniques used for CNS compounds. Techniques include visualization methods, behavioral tests, and quantitative methods.

Keywords: Nanoparticles,CNS, Visualization,Transcytosis,Parameters, uptake mechanism.
\end{abstract}

\section{INTRODUCTION}

Essentially none of the large-molecule pharmaceutics (e.g. peptides, proteins and nucleicacids) can enter the brain, and over $98 \%$ of small molecule drugs cannot enter the braineither ${ }^{1}$. In the past years, several methods to study brain uptake of drugs have been developed. To enhance brain uptake, nanoparticles have been used to target drugs to thebrain. Nanoparticles are different from CNS compounds in size, composition and uptake mechanisms. This has led to different methods and approaches to study brain uptake in vivo. Here we discuss the techniques generally used to measure nanoparticle uptake in addition to the techniques used for CNS compounds.

\section{Drug transport at the blood-brain barrier}

Transport from the blood to the brain is limited by the blood-brain barrier (BBB). The BBBis formed by brain endothelial cells that line the cerebral microvessels. It is supported byother cell types surrounding the endothelium, such as astrocytes and pericytes ${ }^{2}$. These surrounding cells contribute to the induction of many barrier characteristics of theendothelium, such as tight junctions, that closely join the endothelial cells together. Next to being a "physical barrier", the BBB is also a "transport barrier". This aspect is formed byspecific transport proteins and transcytosis mechanisms that mediate the uptake and efflux of molecules. Thirdly, a "metabolic barrier" is formed by the expression of metabolizing enzymes such as peptidases, cytochrome P450 enzymes, and monoamine oxidases ${ }^{3-5}$. All of these barrier functions control and regulate both inward and outward transfer of molecules between blood and the brain.

\section{ROUTES FOR THE TRANSPORT OF MOLECULES ACROSS THE BBB}

Paracellular hydrophilic diffusion:Paracellular transport of hydrophilic molecules is highly restricted by the tight junctions present between brain endothelial cells.

Transcellular lipophilic diffusion: Lipid soluble molecules with molecular weights below $400 \mathrm{Da}$ are able to cross by transcellular lipophilic diffusion, provided that they are not bound to plasma proteins to a high extent, or form a substrate for a transport system at the BBB. Based on physicochemical properties such as molecular weight and hydrogenbonding,predictions can be made whether a compound can cross the $\mathrm{BBB}$ via this route ${ }^{6,7}$.

Receptor mediated endocytosis:For a variety of molecules that are essential for brain function, such as amino acids, glucose, peptides, and proteins, specific endogenous BBB transporters exist. These are expressed at both the luminal and the basolateral membranes of the endothelium ${ }^{8}$. These transporters can be either defined as carriers or receptors.

Carrier mediated transcytosis:Carriers are membranerestricted systems. They are generally responsible for the transport of small molecules with a fixed size and mass smaller than $600 \mathrm{Da}$. Carrier-mediated transcytosisare used for the delivery of nutrients such as glucose, amino acids, and purine bases to the brain. It is substrate selective and only drugs that closely mimic the endogenous carrier substrates will be taken up ${ }^{9}$.

Adsorptive mediated endocytosis:Endocytosis at the BBB is effectuated through adsorption or receptor binding. Adsorptivemediated endocytosis is initiated by the binding of polycationic substances to negative charges on the plasma membrane ${ }^{9}$. Receptor-mediated endocytosis is initiated by the binding of a receptor-specific ligand. Following adsorption or binding, the substance is internalized and transported via the early endosome to the lysosome, or transcytosed to the plasma membrane. The only way for larger molecules and particles such as antibodies, lipoproteins, proteins and nanoparticles to be transported into the brain is via receptor or adsorptivemediated endocytosis ${ }^{10}$, which is different from small molecular weight CNS drugs. When compared to the peripheral endothelium, the cerebral endothelium has a much lower endocytotic and transcytotic activity, making BBB-passage of larger molecules difficult even when 
endocytosis is possible. In pathological conditions, the transport mechanism at the BBB might be up or down regulated ${ }^{1}$.

Efflux pump:Next to these influx systems, many efflux mechanisms exist at the $\mathrm{BBB}$ as well. These include Pglycoprotein, MDR-related protein, ABC transporters, and several others ${ }^{1}$. They restrict entry of molecules into the brain by promoting luminal release of compounds, and are important in removing harmful substances from the brain, thereby reducing toxic side effects of CNS drug metabolites. Substrates for efflux transporters include peptides,lipids, cholesterol, hormones, CNS drugs, and metabolites ${ }^{11}$.

\section{PATHWAYS ACROSS THE BLOOD-BRAIN BARRIER}

After in vivo administration, most CNS drugs will enter the brain in their free form via transcellular diffusion. However, many compounds with psychopharmacological activity do not possess the right physicochemical characteristics to be able to cross the BBB. One possible way to mask these characteristics is to package these compounds in nanoparticles. Nanoparticles are, of course, much larger and are only able to enter the brain endothelial cells via adsorptive or receptor-mediated endocytosis. Subsequent transcytosis to the basal side of the brain endothelial cells is required to enter into the brain parenchyma $^{2}$. After a drug or nanoparticle formulation has been administered, the concentration that can be measured in the brain depends on several factors. Theseinclude: ${ }^{5,13}$

- the plasma concentration-time curve

- the extent of plasma protein binding

- the permeability across the BBB

- the efflux out of the brain by efflux transporters

- the metabolic conversion by enzymes

- the binding to membranes or intracellular sites in the brain

- the continual secretion and drainage of cerebrospinal fluid (CSF)

- brain interstitial fluid (ISF)

\section{IN VIVO TECHNIQUES TO MEASURE COMPOUND PERMEATION INTO THE BRAIN}

A number of in vivo techniques have been developed to measure the uptake of CNS drugs into the brain.

\section{$K_{\text {in }}$ and PS product determination by intravenous injection}

The intravenous injection technique is regarded as the gold standard for brain uptake studies, because it involves fully physiological conditions ${ }^{14,17}$. With this technique, a (radiolabeled) compound is injected intravenously. Blood is sampled at various time points. The main advantages of the iv injection method are the ease of injection, the possibility to simultaneously measure pharmacokinetics, and the fully physiological conditions, enabling all transporters, junction proteins, and enzymes to be present at their physiological concentration.

\section{$K_{\text {in }}$ and PS product determination by in situ perfusion}

The in situ perfusion method complements the iv injection method. It has been originally developed for rats, but it has been expanded for mice, guinea pigs, and rabbits ${ }^{18,20}$. The (c) 2011, JDDT. All Rights Reserved main advantages of the in situ perfusion method are the ability to tailor the perfusion fluid, the constant infusion concentration, and the absence of compound metabolism in other organs ${ }^{17,22}$.

\section{Brain uptake index (BUI)}

The brain uptake index (BUI), represents the relative uptake of a drug compared to a reference substance ${ }^{25,26}$. The reference is freely diffusible across the BBB, such as ${ }^{14} \mathrm{C}$-butanol. The test compound is also radiolabeled, for example with ${ }^{3} \mathrm{H}$. The main advantage of the BUI technique is that it is fast while its main disadvantage is the low sensitivity. Additionally, drugs that are taken up slowly cannot be studied with this method ${ }^{14}$. Examples of BUI values obtained in vivo are $1.4 \%$ for sucrose, and $90 \%$ for caffeine ${ }^{24}$.

\section{Quantitative autoradiography}

Quantitative autoradiography can be used to determine the amount of radioactive test compound in specific regions of the brain, such as stroke-affected areas ${ }^{29}$ or brain tumors ${ }^{30}$, following oral, intravenous or subcutaneous administration to small animals. The strength of quantitative autoradiography lies in the high spatial resolution in the micrometer range ${ }^{14,31}$.

\section{Microdialysis}

Intracerebralmicrodialysis involves the implantation of a microdialysis probe in the brain. The probe, which consists of a semipermeable membrane, is continuously perfused with a physiological solution. The test drug is administered to the animal by the desired route (e.g. oral, intravenous or subcutaneous). Drugs that cross the BBB and enter the brain interstitial fluid can traverse the semipermeable membrane by diffusion into the physiological buffer. The buffer is sampled from the probe, and drug concentration is measured. The concentration in the sample reflects the concentration of free drug in the brain. The main advantage of microdialysis is that brain levels, as well as blood levels of the drug can be determined at many time points in one animal. From these data, pharmacokinetic parameters can be obtained. Drawbacks include the technical difficulties of the implantation, and the fact that highly lipophilic compounds are generally difficult to recover $^{32}$.

\section{Brain/plasma ratio}

Commonly used in the pharmaceutical industry is the brain/plasma ratio ${ }^{33}$. The test drug is administered to the animal by the desired route. At a predetermined time point, the blood is sampled and the brain is taken out. The brain is homogenized and the drug concentration is determined in both brain and plasma. If multiple animals were used for multiple time points, the AUC of both the brain and plasma can be obtained. The brain concentration is then divided by the plasma concentration. This can be the ratio of one time point or the ratio of the $\mathrm{AUCs}^{34}$. The ratio provides a measure of the extent of brain penetration, not of the rate of brain penetration. Usually, the presence of drug remaining in the brain vasculature is not taken into account.

\section{External detection methods}


The techniques described so far involve sampling from the brain. Next to these invasive techniques, several noninvasive external imaging techniques exist, including positron emission tomograpy (PET), and single photon emission computed tomograpy (SPECT). It has been shown that PET can be used to quantitatively measure the PS product ${ }^{35}$. However, PET and SPECT are in general used for imaging of transporters, receptors, inflammation, or tumors in the brain, and not for the uptake of compounds $^{36-38}$.

\section{CNS COMPOUNDS VERSUS NANOPARTICLES}

As the vast majority of potential CNS compounds have limited brain uptake, they may benefit from the use of advanced delivery systems in order to cross the BBB. Nanoparticles have been widely used as drug carriers to increase uptake of such drugs into the brain. The drug is encapsulated in, or associated to the particle, thereby masking its physiochemical characteristics. Particles that have been used include liposomes, solid lipid nanoparticles, nanogels, dendrimers, albumin nanoparticles, and polymeric particles such as poly(lacticco-glycolic acid) (PLGA) and poly(butyl cyanoacrylate) (PBCA) nanoparticles. In many cases, they are combined with targeting ligands on the particle surface to enhance uptake. Ligands can include peptides, proteins, and antibodies.

\section{IN VIVO TECHNIQUES TO MEASURE NANOPARTICLE UPTAKE INTO THE BRAIN}

The design of brain uptake studies of free drugs is generally different from the design of brain uptake studies of nanoparticles, because the size, physicochemical properties, and uptake mechanisms are different. This results in different approaches. The methods that have been used for brain targeting studies using nanoparticles in the past decade are described here.

\section{Visualization methods}

Microscopy is the most widely used qualitative method to study BBB uptake of nanoparticles in vivo.

\section{Fluorescence microscopy}

Fluorescence microscopy is often used for its sensitivity. It requires loading of the nanoparticles with a fluorescent dye $^{51}$, or covalent coupling of the particle building blocks to a dye ${ }^{49}$. Commonly used fluorescent dyes include rhodamine-123, fluorescein, and 6-coumarin. Particles are usually injected iv, and aftersectioning the brain, they can be localized using fluorescence microscopy. The brain endothelium can be visualized by using an endothelium staining marker such as lectin. If such a marker is included in the staining procedure, a distinction can be made between particles that are associated with the brain endothelium, and particles that have crossed into the brain parenchyma $^{53}$. If gene delivery to the brain is of interest, a plasmid expressing a fluorescent protein can be incorporated intothe particle, and gene expression can be assessed by visualizing protein fluorescence in the brain sections $^{48}$.

\section{Electron microscopy}

Electron microscopy is commonly used. With this technique,single particles can be visualized in specific regions of the brain.

\section{Behavioral tests}

Some drugs that act on the CNS are unique compared to drugs that act on any other organ, because of their ability to interfere with brain signaling, and to induce specific behavioral effects. This gives rise to the possibility of not only detecting the level of compound that reaches the brain, but also to determine whether the compound has a pharmacodynamic effect. Drugs that normally do not enter the brain can be encapsulated into nanoparticles, to be transported over the BBB. The effect of the drug in the brain can be read out by monitoring the behavior of the animal, with specifically designed behavioral tests. These tests do not register the whole particle inside the brain, but only the free drug that has been released from the particle, as only the free drug will be able to exert an effect. Drug release from nanoparticles at the site of interest is crucial; therefore behavioral tests are of great value.

\section{Nociceptive tests}

A common model drug that has been used in combination with nanoparticles is the opiate antagonist loperamide. Intracerebral administration of loperamide causes antinociceptive effects. However, after iv injection of loperamide, these effects are not seen. This is due to the efflux membrane transporter P-glycoprotein ${ }^{54}$. Loperamidemolecules that reach the endothelial cells of the blood-brain barrier are rapidly transported back to the blood circulation. Therefore, loperamide is not able to enter into the brain parenchyma, making it a model drug for nociceptive testing. It can be loaded inside nanoparticles ${ }^{50}$, or simply be adsorbed onto the surface of the particles ${ }^{44}$.Loperamide-containing particles that are successfully taken up by the brain, can release their drug content in the brain, causing an analgesic effect of loperamide after ivadministration ${ }^{46}$. Similar to loperamide, the hexapeptidedalargin has been used for this purpose ${ }^{52}$.

\section{Motor function and learning $\&$ memory tests}

Other models that allow for behavioral testing often include induction of disease states in animals, like MPTPinduced Parkinson syndrome. Drugs that treat these syndromes, but normally do not penetrate the BBB in their free form, are candidates for nanoparticle formulation to enhance brain uptake. An example is nerve growth factor $(\mathrm{NGF})^{40}$. NGF has been evaluated for the treatment of neurological diseases such as Alzheimer's disease and Parkinson's disease. However, NGF has a low permeability through the $\mathrm{BBB}$ following intravenous administration $^{39}$.

\section{Quantitative methods}

Quantitative methods to study BBB nanoparticle uptake in vivo, involve determination of pharmacokinetic (PK) parameters, and biodistribution studies. For both of these studies either the particle itself or an entrapped compound is labeled. Most commonly, radioisotopes like ${ }^{3} \mathrm{H},{ }^{14} \mathrm{C}$ or ${ }^{125} \mathrm{I}$ are used. Alternatively, the entrapped compound is quantified by HPLC.

\section{Pharmacokinetic parameters}


In most brain targeting studies, the nanoparticles are administered intravenously. The goal of a pharmacokinetic study is to assess the fraction of the administered dose that is distributed to the brain or is excreted from the body ${ }^{55}$. Nanoparticle and/or drug concentrations are measured in the blood (plasma) and in the brain at several time points after administration. From these measurements, the area under the concentration-time curve (AUC) can be obtained for both blood and brain. Pharmacokinetic models quantify the rate and extent of the distribution by mathematically analyzing these data. In this way,a number of PK parameters can be obtained. Commonly, several of these parameters are determined for either blood ${ }^{43,47}$, brain or both $^{41,42}$. Other organs can also be included in the PK study $^{51}$. In current brain targeting studies, the most widely determined PK parameters in both blood and brain are:

- $\mathrm{C}_{\text {max }}$ : peak concentration

- $\mathrm{T}_{\max }$ : time to reach peak concentration

- AUC: area under the concentration-time curve, from the time of injection $(\mathrm{t}=0)$ to a determined time point $\mathrm{t}$ $\left(\mathrm{AUC}_{(0 \rightarrow \mathrm{t})}\right)$ or extrapolated to infinity $\left(\mathrm{AUC}_{(0 \rightarrow \infty)}\right)$.

- Half-life (t1/2): time it takes for $50 \%$ to be eliminated.

- Mean Residence Time (MRT): the average time a compound remains in the blood or Brain

\section{Biodistribution studies}

For biodistribution studies, the nanoparticles are usually administered intravenously. After administration, the animals are sacrificed at a specific time point, or

\section{REFERENCES}

[1] PardridgeW.M..Blood-brain barrier delivery, Drug Discovery Today.2007, 12, 54-61.

[2] de BoerA.G., van der SandtI.C., GaillardP.J.The role of drug transporters at the blood-brain barrier, Annu Rev PharmacolToxicol. 43 (2003) 629-656.

[3] Ghersi-Egea JF, Leninger-Muller B, Suleman G, Siest G, A. Minn. Localization of drug-metabolizing enzyme activities to blood-brain interfaces and circumventricularorgans, J.Neurochem. 62 (1994) 1089-1096.

[4] Ghersi-Egea JF, Leninger-Muller, Cecchelli R, Fenstermacher JD. Blood-brain interfaces: relevance to cerebral drug metabolism, Toxicol.Lett. 82-83 (1995) 645-653.

[5] Abbott NJ. Prediction of blood-brain barrier permeation in drug discovery from in vivo, in vitro and in silico models, Drug Discov.Today: Technologies. 1 (2004) 337-463.

[6] Cecchelli R, Berezowski V, Lundquist S, M. Culot, M. Renftel, M.P. Dehouck, et al. Modelling of the blood-brain barrier in drug discovery and development, Nat.Rev.DrugDiscov. 6 (2007) 650661.

[7] Pardridge WM. Drug targeting to the brain, Pharm.Res. 24 (2007) 1733-1744.

[8] de BoerA.G., , De Lange EL, van der SandtI.C, Breimer DD. Transporters and the blood-brain barrier (BBB), Int.J.Clin.Pharmacol.Ther. 36 (1998) 14-15.

[9] de BoerA.G, Gaillard PJ. Drug Targeting to the Brain, Annu Rev PharmacolToxicol. 47 (2007) 323-355.

[10] Pardridge WM. The blood-brain barrier: bottleneck in brain drug development, NeuroRx. 2 (2005) 3-14.

[11] Abbott NA, Patabendige AA, Dolman DE, Yusof SR, Begley DJ.Structure and function of the blood-brain barrier, Neurobiol.Dis. 37 (2010) 13-25.

[12] Abbott NJ, Ronnback L, Hansson E. Astrocyte-endothelial interactions at the blood-brain barrier, Nat.Rev.Neurosci. 7 (2006) 41-53.

preferably at several time points. The brain is taken out, along with some of the major organs where the particles are likely to end up, including liver and spleen.

\section{Conclusion and future prospects}

In recent brain targeting studies involving nanoparticles, a wide range of approaches has been used by researchers to determine the extent, rate, and effect of drug and nanoparticle uptake into the brain. Choosing the right method depends on whether you want to obtain quantitative uptake values, visualize the uptake mechanism, or test a drug effect. Each technique has its own advantages and disadvantages, which should be considered before experiments are started. Many parameters like administration route, sampling time points, and the way in which the data are presented should be taken into account. Many studies have shown that nanoparticles can reach the brain. Within the brain microenvironment, targeting of nanoparticles starts with binding and uptake of the particles into the endothelial cells. By using techniques such as brain capillary depletion or electron microscopy, it can be shown that particles have crossed the endothelial barrier, and reached the brain parenchyma. However, the intracellular fate of nanoparticles in vivooften remains unclear. This is due to limitations of in vivo detection methods and techniques. Therefore, intracellular processes such as endocytotic mechanisms currently have to be studied in vitro ${ }^{56,57}$, in an artificial environment. Progress in the field of in vivo detection methods is ongoing ${ }^{58,59}$. In the future, faster and more sensitive detection methods are required to study uptake and trafficking of nanoparticles within the brain.

[13] Abbott NJ, Dolman DE, Patabendige AK. Assays to predict drug permeation across the bloodbrain barrier, and distribution to brain, Curr.DrugMetab. 9 (2008) 901-910.

[14] Bickel U.How to measure drug transport across the blood-brain barrier, NeuroRx. 2 (2005) 15-26.

[15] Pardridge WM. $\log (\mathrm{BB})$, PS products and in silico models of drug brain penetration, Drug Discov.Today. 9 (2004) 392-393.

[16] Pinter GG, Atkins JL, Bell DR. Albumin permeability times surface area (PS) product of peritubular capillaries in kidney, Experientia. 30 (1974) 1045.

[17] Smith QR. A review of blood-brain barrier transport techniques, Methods Mol.Med. 89 (2003) 193-208.

[18] Hervonen H, Steinwall O. Endothelial surface sulfhydryl-groups in blood-brain barrier transport of nutrients, ActaPhysiol.Scand. 121 (1984) 343-351.

[19] MurakamiH., TakanagaH., MatsuoH., OhtaniH., Sawada Y. Comparison of blood-brain barrier permeability in mice and rats using in situ brain perfusion technique, Am.J.Physiol.HeartCirc.Physiol. 279 (2000) H1022-8.

[20] Zlokovic BV, Begley DJ, Djuricic BM, Mitrovic DM. Measurement of solute transport across the blood-brain barrier in the perfused guinea pig brain: method and application to $\mathrm{N}$ methyl-alphaaminoisobutyric acid, J.Neurochem. 46 (1986) 1444 1451 .

[21] Takasato Y, Rapoport SI, Smith QR. An in situ brain perfusion technique to study cerebrovascular transport in the rat, Am.J.Physiol.247 (1984) H484-93.

[22] Smith QR, Allen DD. In situ brain perfusion technique, Methods Mol.Med. 89 (2003) 209-218.

[23] Lockman PR, Koziara J, Roder KE, Paulson J, et al. In vivo and in vitro assessment of baseline blood-brain barrier parameters in the presence of novel nanoparticles, Pharm.Res. 20 (2003) 705 713

[24] Lundquist S, Renftel M, Brillault J, Fenart L, Cecchelli R, M.P. Dehouck. Prediction of drug transport through the blood-brain ISSN: 2250-1177 
barrier in vivo: a comparison between two in vitro cell models, Pharm.Res. 19 (2002) 976-981.

[25] Bonate PL. Animal models for studying transport across the blood-brain barrier, J.Neurosci.Methods. 56 (1995) 1-15.

[26] Oldendorf WH. Measurement of brain uptake of radiolabeled substances using a tritiated water internal standard, Brain Res. 24 (1970) 372-376.

[27] Riant $P$ et al. Effects of the binding of imipramine to erythrocytes and plasma proteins on its transport through the rat blood-brain barrier, J.Neurochem.51 (1988) 421-425.

[28] Pardridge WM, Sakiyama R, Fierer G. Transport of propranolol and lidocaine through the rat bloodbrain barrier. Primary role of globulin-bound drug, J.Clin.Invest. 71 (1983) 900-908.

[29] Knight RA, et al. Estimating bloodand brain concentrations and blood-to-brain influx by magnetic resonance imaging with stepdown infusion of Gd-DTPA in focal transient cerebral ischemia and confirmation by quantitative autoradiography with Gd-[(14)C]DTPA, J.Cereb.Blood Flow Metab. 29 (2009) 1048 1058.

[30] Ferrier $\mathrm{MC}$ et al. Validation of dynamic contrast-enhanced magnetic resonance imaging-derived vascular permeability measurements using quantitative autoradiography in the RG2 rat brain tumor model, Neoplasia. 9 (2007) 546-555.

[31] Lemaire M, Desrayaud S. The priorities/needs of the pharmaceutical industry in drug delivery to the brain, Int.Congr.Ser. 1277 (2005) 32-46.

[32] de LangeE.C., de Boer BA, Breimer DD. Microdialysis for pharmacokinetic analysis of drug transport to the brain, Adv.DrugDeliv.Rev. 36 (1999) 211-227.

[33] Reichel A. Addressing central nervous system (CNS) penetration in drug discovery: basics and implications of the evolving new concept, Chem.Biodivers. 6 (2009) 2030-2049.

[34] Doran A et al. The impact of P-glycoprotein on the disposition of drugs targeted for indications of the central nervous system: evaluation using the MDR1A/1B knockout mouse model, Drug Metab. Dispos. 33 (2005) 165-174.

[35] SchlageterN.L., Carson RE, RapoportS.I.. Examination of blood-brain barrier permeability in dementia of the Alzheimer type with [68Ga]EDTA and positron emission tomography, J.Cereb.BloodFlow Metab. 7 (1987) 1-8.

[36] Urakami T et al. Evaluation of O-[(18)F]fluoromethyl-Dtyrosine as a radiotracer for tumor imaging with positron emission tomography, Nucl.Med.Biol. 36 (2009) 295-303.

[37] Emond P et al.. PE2I: a radiopharmaceutical for in vivo exploration of the dopamine transporter, CNS Neurosci.Ther. 14 (2008) 47-64.

[38] Doorduin J et al. Evaluation of [11C]-DAA1106 for imaging and quantification of neuroinflammation in a rat model of herpes encephalitis, Nucl.Med.Biol. 37 (2010) 9-15.

[39] Poduslo JF, CurranG.L.. Permeability at the blood-brain and blood-nerve barriers of the neurotrophic factors: NGF, CNTF, NT3, BDNF, Brain Res.Mol.Brain Res. 36 (1996) 280-286.

[40] Y. Xie, L. Ye, X. Zhang, W. Cui, J. Lou, T. Nagai, et al. Transport of nerve growth factor encapsulated into liposomes across the blood-brain barrier: in vitro and in vivo studies, J.Control.Release. 105 (2005) 106-119.

[41] H. Chen, L. Tang, Y. Qin, Y. Yin, J. Tang, W. Tang, et al. Lactoferrin-modified procationic liposomes as a novel drug carrier for brain delivery, Eur.J.Pharm.Sci. 40 (2010) 94-102.

[42] J.X. Wang, X. Sun, Z.R. Zhang. Enhanced brain targeting by synthesis of 3',5'-dioctanoyl-5-fluoro-2'-deoxyuridine and incorporation into solid lipid nanoparticles, Eur.J.Pharm.Biopharm. 54 (2002) 285-290.

[43] V. Mishra, S. Mahor, A. Rawat, P.N. Gupta, P. Dubey, K. Khatri, et al. Targeted brain delivery of AZT via transferrin anchored pegylated albumin nanoparticles, J.Drug Target. 14 (2006) 45-53.

[44] K. Michaelis, M.M. Hoffmann, S. Dreis, E. Herbert, R.N. Alyautdin, M. Michaelis, et al. Covalent linkage of apolipoprotein e to albumin nanoparticles strongly enhances drug transport into the brain, J.Pharmacol.Exp.Ther. 317 (2006) 1246-1253.

[45] A. Zensi, D. Begley, C. Pontikis, C. Legros, L. Mihoreanu, S. Wagner, et al. Albumin nanoparticles targeted with Apo E enter the CNS by transcytosis and are delivered to neurones, J.Control.Release. 137 (2009) 78-86.

[46] K. Ulbrich, T. Hekmatara, E. Herbert, J. Kreuter. Transferrinand transferrin-receptor-antibodymodified nanoparticles enable drug delivery across the blood-brain barrier (BBB), European Journal of Pharmaceutics and Biopharmaceutics. 71 (2009) 251 256.

[47] J. Lode, I. Fichtner, J. Kreuter, A. Berndt, J.E. Diederichs, R. Reszka. Influence of surface-modifying surfactants on the pharmacokinetic behavior of 14C-poly (methylmethacrylate) nanoparticles in experimental tumor models, Pharm.Res. 18 (2001) 1613-1619.

[48] R. Huang, W. Ke, L. Han, Y. Liu, K. Shao, C. Jiang, et al. Lactoferrin-modified nanoparticles could mediate efficient gene delivery to the brain in vivo, Brain Res.Bull. 81 (2010) 600-604.

[49] L. Costantino, F. Gandolfi, G. Tosi, F. Rivasi, M.A. Vandelli, F. Forni. Peptide-derivatized biodegradable nanoparticles able to cross the blood-brain barrier, J.Control.Release. 108 (2005) 84-96.

[50] G. Tosi, L. Costantino, F. Rivasi, B. Ruozi, E. Leo, A.V. Vergoni, et al.Targeting the central nervous system: In vivo experiments with peptide-derivatized nanoparticles loaded with Loperamide and Rhodamine-123, Journal of Controlled Release. 122 (2007) 1-9.

[51] F. Xu, W. Lu, H. Wu, L. Fan, X. Gao, X. Jiang. Brain delivery and systemic effect of cationic albumin conjugated PLGA nanoparticles, J.Drug Target. 17 (2009) 423-434.

[52] J. Kreuter, D. Shamenkov, V. Petrov, P. Ramge, K. Cychutek, C. Koch-Brandt, et al. Apolipoproteinmediated Transport of Nanoparticle-bound Drugs Across the Blood-Brain Barrier, Journal of Drug Targeting. 10 (2002) 317.

[53] C.K. Weiss, M.V. Kohnle, K. Landfester, T. Hauk, D. Fischer, J. Schmitz-Wienke, et al. The first step into the brain: uptake of NIO-PBCA nanoparticles by endothelial cells in vitro and in vivo, and direct evidence for their blood-brain barrier permeation, ChemMedChem. 3 (2008) 1395-1403.

[54] A.J. Sadeque, C. Wandel, H. He, S. Shah, A.J. Wood.Increased drug delivery to the brain by Pglycoprotein inhibition, Clin.Pharmacol.Ther. 68 (2000) 231-237.

[55] J.E. Riviere. Pharmacokinetics of nanomaterials: an overview of carbon nanotubes, fullerenes and quantum dots, Wiley Interdiscip.Rev.NanomedNanobiotechnol. 1 (2009) 26-34.

[56] J. Chang, Y. Jallouli, M. Kroubi, X. Yuan, W. Feng, C. Kang, et al. Characterization of endocytosis of transferrin-coated PLGA nanoparticles by the blood-brain barrier, Int.J.Pharm. 379 (2009) 285-292.

[57] Y. Jallouli, A. Paillard, J. Chang, E. Sevin, D. Betbeder. Influence of surface charge and inner composition of porous nanoparticles to cross blood-brain barrier in vitro, Int.J.Pharm. 344 (2007) 103-109.

[58] R.A. de Kemp, F.H. Epstein, C. Catana, B.M. Tsui, E.L. Ritman. Small-animal molecular imaging methods, J.Nucl.Med.51 Suppl 1 (2010) 18S-32S.

[59] D.M. Chudakov, M.V. Matz, S. Lukyanov, K.A. Lukyanov. Fluorescent proteins and their applications in imaging living cells and tissues, Physiol.Rev. 90 (2010) 1103-1163. 